\title{
INDIRECT EXCHANGE IN MAGNETIC SEMICONDUCTORS. III. EFFECT OF ALLOYING*
}

\author{
M. MatLaK \\ Institute of Physics, Silesian University, Uniwersytecka 4, 40-007 Katowice, Poland
}

(Received April 17, 1990; in revised form August 9, 1990)

We apply the extended $s-f$ model to magnetic pseudobinary alloys. We calculate the Curie temperature and magnetic moment in the paramagnetic state as functions of concentration $x$. The calculated quantities are in good agreement with the quantities experimentally measured for ${E u_{1-x}}_{G_{1}} \mathrm{Gd}_{x}$, $\mathrm{Eu}_{1-x} \mathrm{Gd}_{x} \mathrm{Se}, \mathrm{Re}_{x} \mathrm{Gd}_{1-x} \mathrm{Al}_{2}(\mathrm{Re}=\mathrm{Dy}, \mathrm{Ho}, \mathrm{Er}, \mathrm{La}, \mathrm{Lu}, \mathrm{Y}), \mathrm{Gd}\left(\mathrm{Al}_{1-x} \mathrm{Me}_{x}\right)_{2}$ $(\mathrm{Me}=\mathrm{Cu}, \mathrm{Ag}, \mathrm{Pd}, \mathrm{In}, \mathrm{Sn}), \ldots$

PACS numbers: 75.10.-b, 75.30.-Cr, 75.20.-En, 75.50.-y

\section{Introduction}

In the papers $[1,2]$ the extended $s-f$ model has been succesfully used to explain the observed direct current (d.c.) conductivity dependence on temperature and the Curie temperature dependence on applied pressure for EuO. The model can be, however, applied to a more wide class of materials as e.g. normal-valent or intermediate-valent magnetic rare earth compounds and alloys. One of the most striking properties of the extended $s-f$ model is "self-production" of the indirect exchange interaction between $4 f$ electrons even if the direct $4 f-4 f$ exchange is absent in the model $[1,2]$.

In the present paper we consider an alloy whose constituents can be described by the extended $s-f$ model as e.g. disordered magnetic rare earth alloys [3-11]. We calculate the Curie temperature and the magnetic moment in the paramagnetic state as functions of the concentration of the alloy.

*Supported by CPBP 01.12. 


\section{Magnetic susceptibility}

The Hamiltonian of the extended $s-f$ model (cf. e.g. [2] and the papers cited therein) has the form:

$$
H=H_{f}+H_{d}+H_{f-d}+H_{z}+H_{N}
$$

where

$$
\begin{gathered}
H_{f}=E_{f} \sum_{i, \sigma} n_{i, \sigma}^{f}+U_{f} \sum_{i} n_{i, \uparrow}^{f} n_{i, \downarrow}^{f}, \\
H_{d}=t_{0} \sum_{i, \sigma} n_{i, \sigma}^{d}+\sum_{i, j, \sigma} t_{i, j} d_{i, \sigma}^{+} d_{j, \sigma} \\
H_{f-d}=-\frac{g}{2} \sum_{i}\left(f_{i, \uparrow}^{+} f_{i, \downarrow} d_{i, \downarrow}^{+} d_{i, \uparrow}+f_{i, \downarrow}^{+} f_{i, \uparrow} d_{i, \uparrow}^{+} d_{i, \downarrow}\right. \\
\left.+\frac{1}{2}\left(n_{i, \uparrow}^{f}-n_{i, \downarrow}^{f}\right)\left(n_{i, \uparrow}^{d}-n_{i, \downarrow}^{d}\right)\right)+V \sum_{i, \sigma}^{n}\left(f_{i, \sigma}^{+} d_{i, \sigma}+d_{i, \sigma}^{+} f_{i, \sigma}\right) \\
H_{z}=-\mu_{\mathrm{B}} \mathcal{H} \sum_{i}\left(n_{i, \uparrow}^{f}-n_{i, \downarrow}^{f}\right)-\mu_{\mathrm{B}} \mathcal{H} \sum_{i}\left(n_{i, \uparrow}^{d}-n_{i, \downarrow}^{d}\right),
\end{gathered}
$$

and

$$
H_{N}=-\mu \sum_{i, \sigma}\left(n_{i, \sigma}^{f}+n_{i, \sigma}^{d}\right) .
$$

The term $H_{f}$ describes the $4 f$ electrons of the magnetic rare earth, $E_{f}$ is the position of the atomic $4 f$ level and $U_{f}$ is the Coulomb repulsion. $H_{d}$ describes the conduction electrons and $H_{f-d}$ represents the $s-f$ interaction between $4 f$ and conduction electrons and the hybridization responsible for the valence fluctuations. The term $H_{z}$ describes the interaction of the $4 f$ and conduction electron spins with the external magnetic field $\mathcal{H}$ and $\mu$, as usually, means the chemical potential of the system.

Using the formulae (24) and (25) from [2] $\left(U_{f} \rightarrow \infty\right)$ it is easy to find the total susceptibility:

$$
\chi=\chi^{(f)}+\chi^{(d)}=\frac{2 A \mu_{\mathrm{B}}^{2}}{1-\frac{T_{c}}{T}},
$$

where $A$ is a function of the model parameters and temperature $\left(A=A\left(E_{f}, g, V, W\right.\right.$, $\mu, T), W$ is the bandwidth of the conduction band) and $T_{C}$ is given by the implicite equation of the form:

$$
T_{\mathrm{C}}=f\left(E_{f}, g, V, W, \mu, T_{\mathrm{C}}\right)
$$

and determines the Curie temperature of our system. Equation (8) coincides with the equation for $T_{\mathrm{C}}$ used in [2]. Both $A$ and the equation for $T_{\mathrm{C}}$ are very complicated and cannot be presented here.

The equation for the chemical potential has the same form here as in [2]:

$$
2\left(<n_{\uparrow}^{f}>+<n_{\uparrow}^{d}>\right)=1 .
$$


In Ref. [2] we have shown that our system, where no direct $4 f-4 f$ exchange interaction is present, behaves like an effective Heisenberg ferromagnet with effective exchange integral of indirect origin. For such an effective medium it is easy to find the magnetic susceptibility:

$$
\chi=\frac{\frac{1}{3} \beta \mu_{\mathrm{eff}}^{2}}{1-\frac{T_{Q}}{T}} .
$$

The comparison between (10) and (7) gives the effective magnetic moment:

$$
\mu_{\mathrm{eff}}=\mu_{\mathrm{B}} \sqrt{6 k T A} \text {. }
$$

Solving numerically the system (8) and (9) we can calculate (11). The result of such a calculation is vizualized in Fig. 1, where, for simplicity, we show the dependence of the magnetic moment at $T=T_{\mathrm{C}}$ versus the position of the $4 f$ level $E_{f}$ for different hybridization parameters $V$. It is interesting to note that the ferromagnetic area in the phase diagram of the model where $T_{\mathrm{C}} \neq 0$ coincides with the area where $\mu_{\text {eff }}\left(T=T_{\mathrm{C}}\right) \neq 0$ (see Fig. 1 in [1]). In the paramagnetic state $\mu_{\text {eff }}\left(T>T_{\mathrm{C}}\right)>0$.

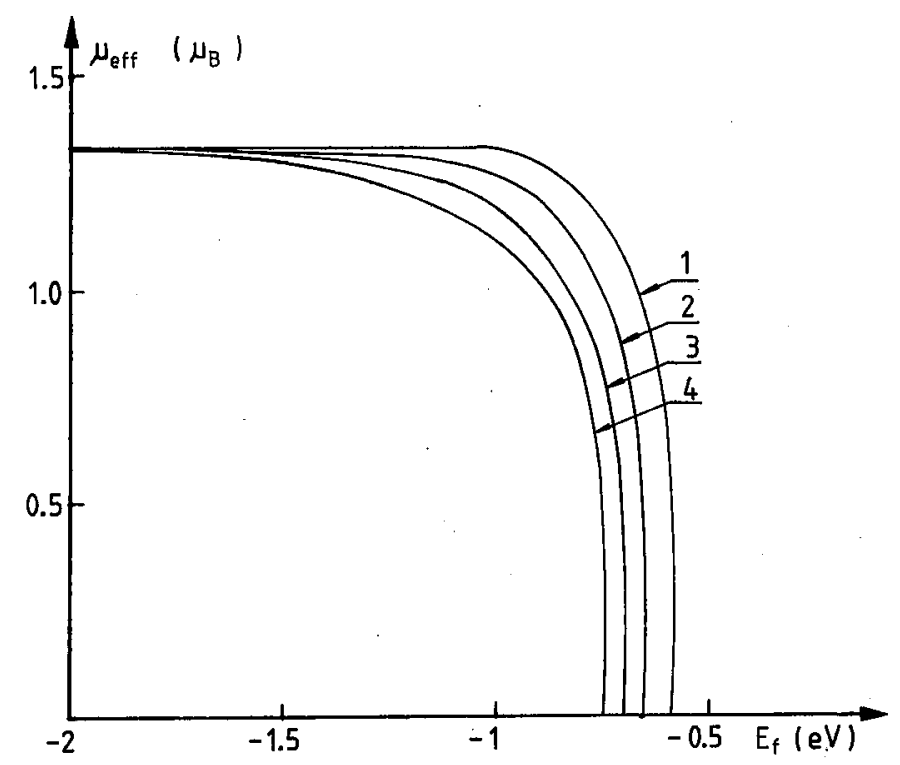

Fig. 1. Magnetic moment in the paramagnetic state vs. position of the $4 f$ level $E_{f}$ for different hybridization $V[\mathrm{eV}]:$ 1) $V=0.1,2) V=0.2,3) V=0.3,4) V=0.4$. 


\section{Effect of alloying}

The general expression for the magnetic moment (11) and the equation for the Curie temperature (8) can easily be used to consider an alloy $\operatorname{Re}_{1-x}^{(1)} \operatorname{Re}_{x}^{(2)} \ldots$, where two magnetic rare earth elements $\operatorname{Re}^{(1)}, \operatorname{Re}^{(2)}$ are magnetic, and to both of them the Hamiltonian (1) can be applied. In this case we have to consider two extreme regimes for the parameters of the model describing the alloy

$$
\begin{aligned}
& x=0: E_{f}=E_{f}^{(1)}, t_{0}=t_{0}^{(1)}, t_{i j}=t_{i j}^{(1)}, W=W^{(1)}, g=g^{(1)}, V=V^{(1)}, \\
& x=1: E_{f}=E_{f}^{(2)}, t_{0}=t_{0}^{(2)}, t_{i j}=t_{i j}^{(2)}, W=W^{(2)}, g=g^{(2)}, V=V^{(2)} .
\end{aligned}
$$

It is easy to see that the equation for the Curie temperature (8) and the expression for the magnetic moment (11) should be replaced by

$$
\begin{gathered}
T_{\mathrm{C}}=(1-x) T_{\mathrm{C}}\left(p^{(1)}, \mu\right)+x T_{\mathrm{C}}\left(p^{(2)}, \mu\right), \\
\mu_{\mathrm{eff}}=\mu_{\mathrm{B}} \sqrt{6 k T}\left((1-x) \sqrt{A^{(1)}\left(p^{(1)}, \mu\right)}+x \sqrt{\left.A^{(2)}\left(p^{(2)}, \mu\right)\right)},\right.
\end{gathered}
$$

where $p^{(1,2)}$ means the parameter set (12) or (13). The equation which should be used to determine the chemical potential of the alloy takes the form:

$$
2\left((1-x)\left(<n_{\uparrow}^{f}>^{(1)}+<n_{\uparrow}^{d}>^{(1)}\right)+x\left(<n_{\uparrow}^{f}>^{(2)}+<n_{\uparrow}^{d}>^{(2)}\right)\right)=a+b x,
$$

where $\left.\left\langle n_{\uparrow}^{f, d}\right\rangle\right\rangle^{(1,2)}$ are the averaged occupation numbers dependent on the parameter set $p^{(i)}(i=1,2), a$ and $b$ are the parameters determining the number of valence electrons of the alloy

$$
\left((1-x) a^{(1)}+x a^{(2)}=a^{(1)}+\left(a^{(2)}-a^{(1)}\right) x \equiv a+b x\right) .
$$

After solving the set of equations (14) and (16) we get $T_{\mathrm{C}}=T_{\mathrm{C}}(x)$ and from (15) the effective magnetic moment $\mu_{\text {eff }}=\mu_{\text {eff }}(x)$. The results are depicted in Fig. 2(a), (b), where the total number of conduction electrons (rhs of (16)) has been taken to be an increasing or decreasing function of $x$. The dependence of the Curie temperature on the concentration $x$, presented in Fig. 2(a) is very similar to the measured for $\mathrm{Eu}_{1-x} \mathrm{Gd}_{x} \mathrm{~S}, \mathrm{Eu}_{1-x} \mathrm{Gd}_{x} \mathrm{Se}$ [3-5], $\operatorname{Re}_{1-x} \mathrm{Gd}_{x} \mathrm{Al}_{2}$ (Re= Dy, Ho, $\mathrm{Er}, \mathrm{La}, \mathrm{Lu}, \mathrm{Y})$ [6-10], $\mathrm{Gd}\left(\mathrm{Al}_{1-x} \mathrm{Me}_{x}\right)_{2} \cdot(\mathrm{Me}=\mathrm{Cu}, \mathrm{Ag}, \mathrm{Pd}, \mathrm{In}, \mathrm{Sn})$ [9-11]. Also the magnetic moment as a function of $x$ (Fig. 2(b)) behaves very similar to the measured one in [11].

An alternative treatment of the magnetic rare earth alloys has been discussed in [12]. The application of this formalism has been given in [13] and [14] for $\mathrm{Gd}_{1-x} \operatorname{Re}_{x} \mathrm{Al}_{2}(\mathrm{Re}=\mathrm{Dy}, \mathrm{Ho}, \mathrm{Er})$ and in [8] for $\operatorname{Re}_{1-x} \mathrm{Gd}_{x} \mathrm{Al}_{2}(\mathrm{Re}=\mathrm{La}, \mathrm{Lu}, \mathrm{Y})$. The use of the multiband $s-f$ model in application to the magnetic properties of $\mathrm{Gd}\left(\mathrm{Al}_{1-x} \mathrm{Me}_{x}\right)_{2}(\mathrm{Me}=\mathrm{Cu}, \mathrm{Ag}, \mathrm{Pd}, \mathrm{In}, \mathrm{Sn})$ has been given in [15]. 

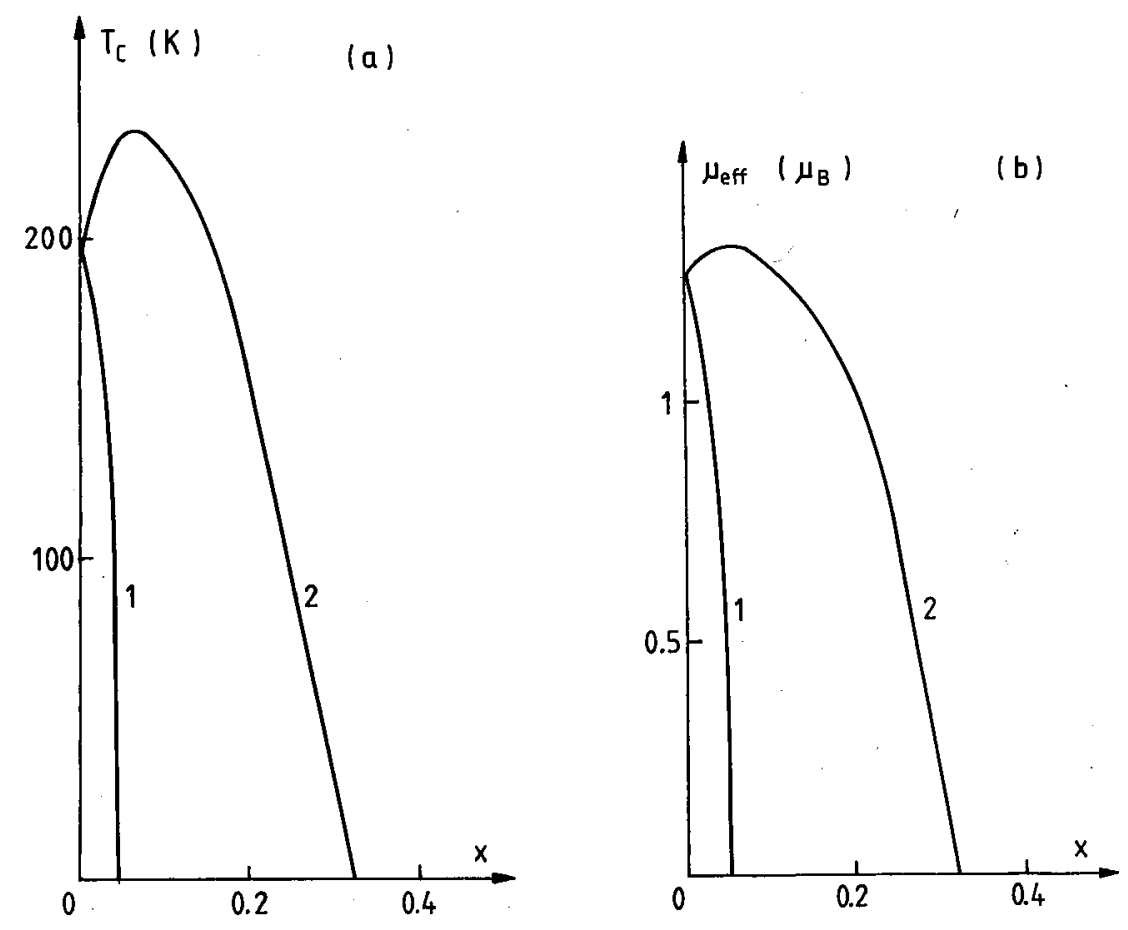

Fig. 2(a). Plot of the Curie temperature $T_{\mathrm{C}}$ vs. concentration $x$. The parameters (in eV): $\left(p^{(1)}\right) E_{f}^{(1)}=-1.5, t_{0}^{(1)}=0, W^{(1)}=2, g^{(1)}=0.3, V^{(1)}=0.3$; $\left(p^{(2)}\right) E_{f}^{(2)}=-1.6, t_{0}^{(2)}=-0.5, W^{(2)}=2, g^{(2)}=0.2, V^{(2)}=0.2$. 1) $a=b=1,2) a=1, b=-1$.

(b) Magnetic moment in the paramagnetic state versus concentration $x$. The parameters are the same as in (a). 


\section{References}

[1] M. Matlak, Solid State Commun. 65, 1575 (1988).

[2] M. Matlak, Acta Phys. Pol. A75, 593, 609 (1989).

[3] F. Holtzberg, T.R. McGuire, S. Methfessel, J.C. Suits, Phys. Rev. Lett. 13, 18 (1964).

[4] S. Methfessel, Z. Angew. Math. Phys. 18, 414 (1965).

[5] F. Holtzberg, T.R. McGuire, S. Methfessel, J. Appl. Phys. 37, 976 (1966).

[6] K.H. Buschow, Rep. Prog. Phys. 42, 1373 (1979).

[7] A. Chełkowski, E. Talik, G. Wnętrzak, J. Phys. F 13, 483 (1983).

[8] A. Ślebarski, E. Zipper, J. Auleytner, J. Phys. F 13, 2643 (1983).

[9] A. Chetkowski, G. Wnętrzak, Acta Phys. Pol. A68, 485 (1985).

[10] A. Ślebarski, J. Magn. Magn. Maler. 66, 107 (1987).

[11] A. Chelkowski, G. Wnętrzak, J. Less-Common Met. 111, 157 (1985).

[12] B. Kozarzewski, Acta Phys. Pol. A59, 603 (1981).

[13] K. Bialas-Borgiel, E. Zipper, W. Borgiel, Acta Phys. Pol. A60, 701 (1981).

[14] K. Witański, Phys. Status Solidi B 120, 149 (1983); 131, 129 (1985).

[15] M. Matlak, J. Zieliński, Acta Phys. Pol. A78 929 (1990). 